\title{
Correction to: Draft Genome Sequence of Cyclohexylamine-Degrading Strain Acinetobacter sp. YT-02 Isolated
}

\author{
Da-Zhong Yan ${ }^{1} \cdot$ Ya-Ting Gan ${ }^{1} \cdot$ Hui Zhou ${ }^{1} \cdot$ Jun Liu ${ }^{1} \cdot$ Xin Li $^{1}$
}

Published online: 24 March 2018

(C) Springer Science+Business Media, LLC, part of Springer Nature 2018

\section{Correction to: Curr Microbiol (2018) 75:284-287 \\ https://doi.org/10.1007/s00284-017-1377-9}

The original version of this article unfortunately contained a mistake in Fig. S1 of supplementary material. It is corrected with this erratum.

Electronic Supplementary Material The online version of this article (https://doi.org/10.1007/s00284-018-1470-8) contains supplementary material, which is available to authorized users.

The original article can be found online at https://doi.org/10.1007/ s00284-017-1377-9.

Da-Zhong Yan

yandz6808@163.com

1 School of Biology and Pharmaceutical Engineering,

Wuhan Polytechnic University, No.68 Xuefu South Road,

Changqing Garden, Wuhan 430023, Hubei Province, China 